\title{
Activity of meropenem, against Gram-positive bacteria
}

\author{
F. H. Kayser, G. Morenzoni, A. Strässle and K. Hadorn \\ Institute of Medical Microbiology, University of Zürich, CH-8028 Zürich, Switzerland
}

\begin{abstract}
A new carbapenem antibiotic, meropenem, was shown to be active against a large number of Gram-positive bacteria. The drug inhibited penicillinase-positive and -negative, methicillin-susceptible staphylococci equally well. Among the comparative antimicrobials examined, only $\mathrm{N}$-formimidoyl-thienamycin (imipenem) was two to four times more active than meropenem. Compared with vancomycin or methicillin, meropenem was 10-20 times more active. Strains of 11 species of streptococci were highly susceptible to meropenem; the geometric mean MICs of the drug for these species ranged from 0.01 to $0.04 \mathrm{mg} / \mathrm{l}$. The agent, however, only had moderate activity against Enterococcus faecalis (mean MIC $5 \mathrm{mg} / \mathrm{l}$ ) and Ent. faecium (mean MIC $11.6 \mathrm{mg} / \mathrm{l})$. Among Corynebacterium jeikeium, strains were encountered that showed susceptibility to meropenem but resistance to imipenem and other $\beta$ lactams. Strains of other corynebacteria, Rhodococcus equi, Erysopelothrix rhusiopathiae, Listeria monocytogenes, and Bacillus spp. all were highly susceptible to meropenem (mean MICs $0-04-0-17 \mathrm{mg} / 1$ ). Although methicillin-resistant staphylococci were inhibited by concentrations of $1-2 \mathrm{mg} / \mathrm{l}$ of meropenem in agar dilution tests, such strains showed heteroresistance in population studies, as is typical for all $\beta$-lactam antibiotics. In addition, the biochemical correlate of methicillin-resistance, penicillin-binding protein 2', showed low affinity for meropenem, similar to that for imipenem. Meropenem was as bactericidal as imipenem and comparative bactericidal antimicrobials in killing-curve experiments. Strains of Ent. faecium, C. jeikeium. and $L$. monocytogenes were killed at a slower rate than streptococci or staphylococci.
\end{abstract}

\section{Introduction}

This study was undertaken to compare the in-vitro activity of meropenem with those of imipenem and various other antimicrobials against Gram-positive organisms. Special emphasis in our study was given to the bactericidal activity of meropenem, which was determined in killing-curve experiments.

\section{Materials and methods}

\section{Bacteria}

The bacteria used were isolated from clinical material and were identified according to standard procedures. Unusual organisms of no or only low pathogenic potential were included in order to obtain an overview of the Gram-positive spectrum of the drug. The strains Staphylococcus aureus ATCC 29213, Escherichia coli ATCC 25922, and Pseudomonas aeruginosa ATCC 27853 were used for quality control. 


\section{Antibiotics}

Stock solutions of meropenem and ciprofloxacin were prepared in sterile distilled water. Stock solutions of the other drugs were prepared as recommended by the NCCLS (1987). Stocks of meropenem and imipenem were freshly prepared for each experiment. The other stock solutions were stored at $-80^{\circ} \mathrm{C}$. All drugs were kindly provided as laboratory powders by the respective pharmaceutical companies.

\section{Minimal inhibitory concentration tests}

MICs were determined by an agar dilution procedure (NCCLS, 1985, 1987) with Mueller-Hinton (MH) agar (BBL) for staphylococci and Mueller-Hinton agar supplemented with $7 \%$ human blood for all other organisms. Staphylococci were tested on Mueller-Hinton agar as well as on Mueller-Hinton agar supplemented with $4 \% \mathrm{NaCl}$ (Jorgensen, 1986) in order to detect methicillin-resistance. MICs of methicillin were read after incubation at $35^{\circ} \mathrm{C}$ for exactly $24 \mathrm{~h}$. Methicillin-resistance was suggested when growth was observed on the plate containing $8 \mathrm{mg} /$. MICs of the other $\beta$-lactam antibiotics were then compared in order to make a final decision about the presence of methicillin-resistance.

\section{Bactericidal tests}

Selected staphylococcal strains grown on blood agar were suspended in MH broth to approximately $10^{6}-10^{7} \mathrm{cfu} / \mathrm{ml}$. All other organisms were taken from blood agar and suspended in $\mathrm{MH}$ broth supplemented with cations and $2.5 \%$ lysed horse blood (NCCLS, 1987). Antibiotics were added in concentrations of $\frac{1}{2}, 1,2,4$ and 8 times the MIC, respectively. One culture served as growth control. Incubation was carried out at $37^{\circ} \mathrm{C}$ in a water bath. At appropriate time intervals, samples were withdrawn and plated undiluted or in appropriate dilutions on to blood agar plates to determine the viable count. When necessary, $\beta$-lactam antibiotics in samples were inactivated by adding $12.5 \mathrm{U} / \mathrm{ml}$ of a mixture of four penicillinases (Porton Products Ltd, Salisbury, Wiltshire, UK), and incubating the samples at room temperature for at least $10 \mathrm{~min}$ before plating. Inactivation of $\beta$-lactams including the carbapenems was confirmed by a qualitative disc diffusion test.

\section{Population studies}

Populations of methicillin-resistant and -susceptible staphylococci were analysed by disaggregation of overnight broth cultures (briefly, by ultrasonication at $20 \mathrm{kHz}$ ) and surface inoculation of appropriate dilutions on L-agar (Berger-Bāchi, Strässle \& Kayser, 1986) containing increasing amounts of meropenem. Colony-forming units were counted after $48 \mathrm{~h}$ of incubation at $35^{\circ} \mathrm{C}$.

\section{Penicillin-binding proteins}

Affinity of $\beta$-lactam antibiotics for penicillin-binding proteins (PBPs) prepared from cell membranes was examined in competition assays with radioactive benzylpenicillin, by a procedure slightly modified from that described recently by Berger-Bächi et al. (1986). Membrane preparations were incubated for $10 \mathrm{~min}$ at $37^{\circ} \mathrm{C}$ in the presence of 0 , 
$0-5,5,50$ and $500 \mathrm{mg} / \mathrm{l}$ of either meropenem or imipenem before reaction with $10 \mathrm{mg} / \mathrm{l}$ ${ }^{3}[\mathrm{H}]$-labelled benzylpenicillin-ethyl-piperidinium salt ( $25 \mathrm{Ci} / \mathrm{mmol}, \mathrm{Merck}, \mathrm{USA}$ ) for $10 \mathrm{~min}$ at $37^{\circ} \mathrm{C}$. $50 \mu \mathrm{g}$ protein was loaded per slot. PBPs were separated by SDS polyacrylamide gel electrophoresis. The acrylamide to $\mathrm{NN}^{\prime}$ methylene bis-acrylamide ratio was $3.75-0.1 \%$ in the stacking gel and $10-0-13 \%$ in the running gel. PBPs were detected by scintillation fluorography with NEN enhancer (New England Nuclear, USA) and exposing the gels at $-70^{\circ} \mathrm{C}$ to Fuji RX1 film.

\section{Results}

\section{Comparison of meropenem with other antimicrobials against Gram-positive bacteria}

Table I summarizes the MICs of meropenem and other drugs for Gram-positive bacteria. Meropenem showed excellent activity against methicillin-susceptible staphylococci, whether they produced penicillinase or not. The drug was 12-14 times more active than methicillin. Imipenem was three to five times more active than meropenem against these strains. All of the eleven streptococcal species tested were highly susceptible to meropenem and to imipenem. Both drugs, however, exhibited only moderate activity against Enterococcus faecalis, and were of rather low activity against Ent. faecium.

Corynebacterium jeikeium has been associated with infections in immunosuppressed patients (Young et al., 1981). The organism has also been isolated occasionally from cases of peritonitis in patients undergoing continuous ambulatory peritoneal dialysis (Pierard et al., 1983). Often, these bacteria have been found to be resistant to many antimicrobials including the aminoglycosides and $\beta$-lactam antibiotics (Gill et al., 1981). This situation has been found also with the collection of strains examined in this study. However, some strains that were highly resistant to imipenem (MICs > $128 \mathrm{mg} / \mathrm{l}$ ) were susceptible to meropenem (MICs $2-8 \mathrm{mg} / \mathrm{l}$ ). Other corynebacteria generally were highly susceptible to both carbapenems. Highly susceptible, also, were the Listeria monocytogenes strains examined. MICs of meropenem for the NCCLS quality control strains determined in agar dilution tests were the same on either Mueller-Hinton agar or Mueller-Hinton agar supplemented with $7 \%$ human blood. The range of MICs for Staph. aureus ATCC 29213 was $0-06-0-12 \mathrm{mg} /$, that for Esch. coli ATCC $259220.016-0.03$, and that for P. aeruginosa $0.25-1 \mathrm{mg} / 1$.

\section{Results with methicillin-resistant staphylococci}

Meropenem and imipenem showed some activity against methicillin-resistant staphylococci, when tested in agar dilution with an inoculum of $10^{4} \mathrm{cfu}$ per spot (see Table I). In tests with a higher inoculum, i.e. in macrobroth dilution tests, MICs of these carbapenems were considerably higher (data not shown). This phenomenon resulted from the heterogeneous resistance to $\beta$-lactam antibiotics in such strains (Kayser et al., 1972). Figure 1 shows the population analysis of four isogenic Staph. aureus strains on meropenem. Strain BB255 was the susceptible parent strain. Strain BB270 was obtained by introducing the methicillin resistance determinant mec from a wild type strain into BB255. Two main resistance levels were recognizable: a low basal level, displayed by all cells, and a second higher, but heterogeneous, level shown here by one in $10^{3}$ cells. The phenotypically susceptible strain BB308, derived from strain BB270 by Tn551-insertion of $\Omega 2003$ into the chromosome, although harbouring the intact 
F. H. Kayser et al.

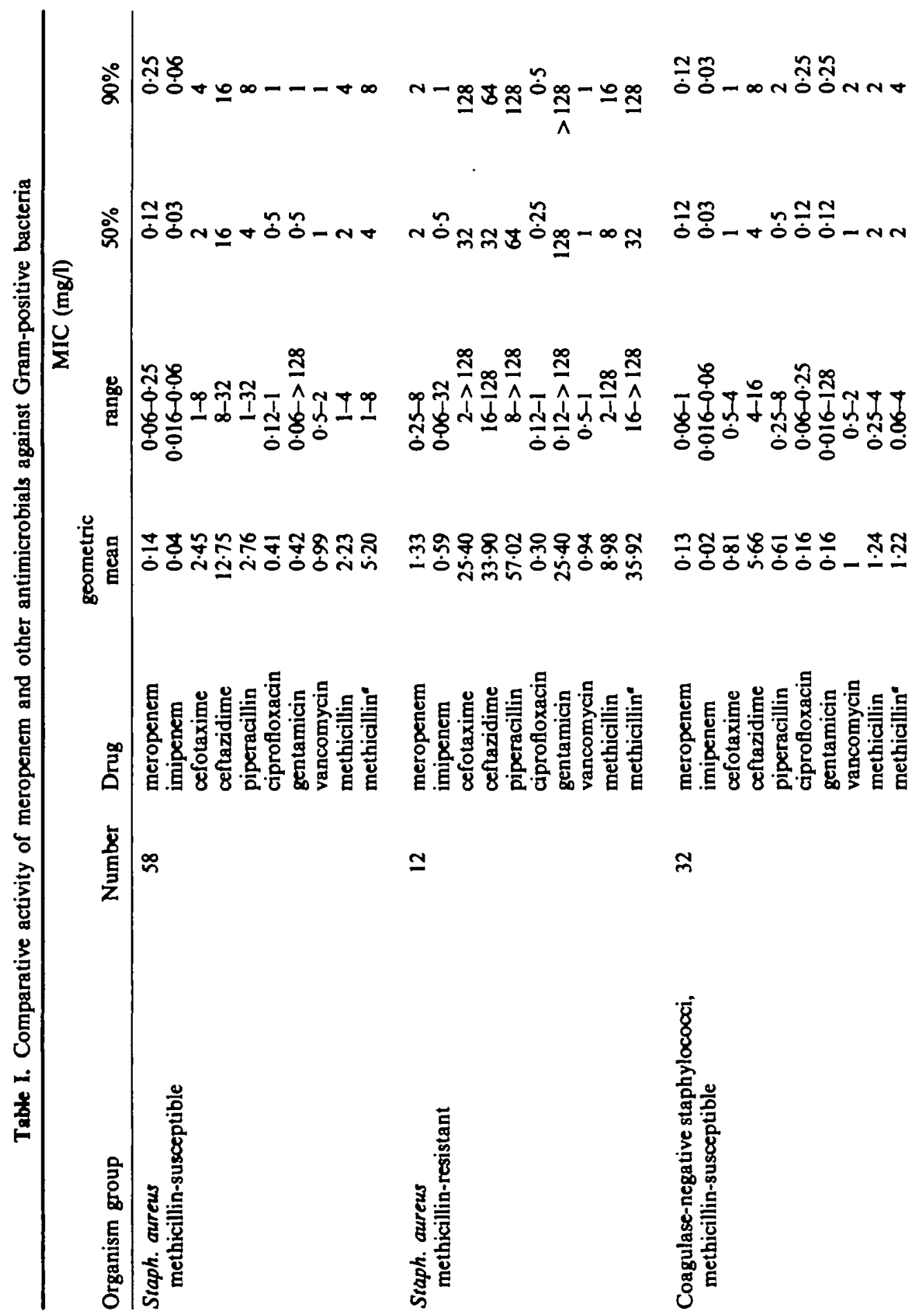




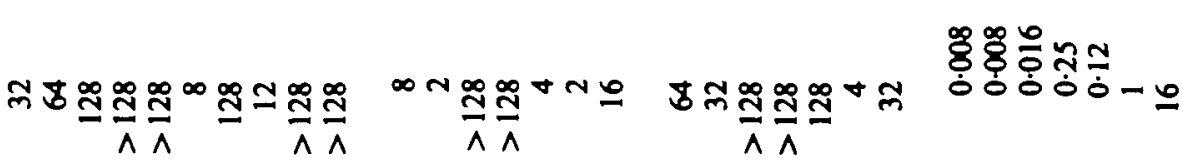

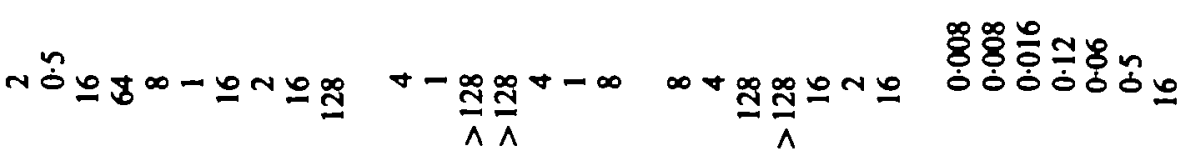

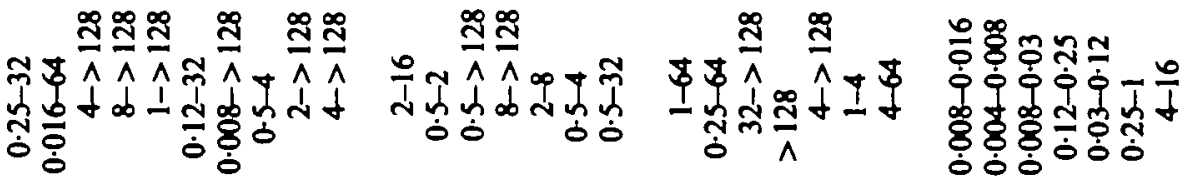

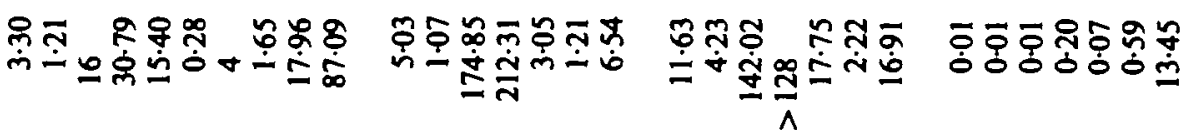

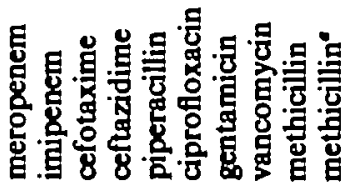

$\stackrel{\infty}{2}$

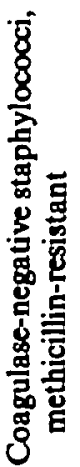

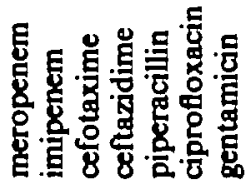
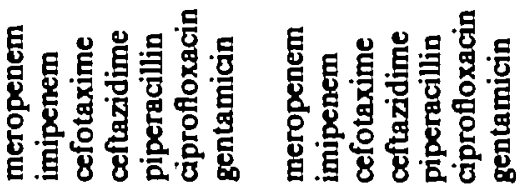

g

2

오 


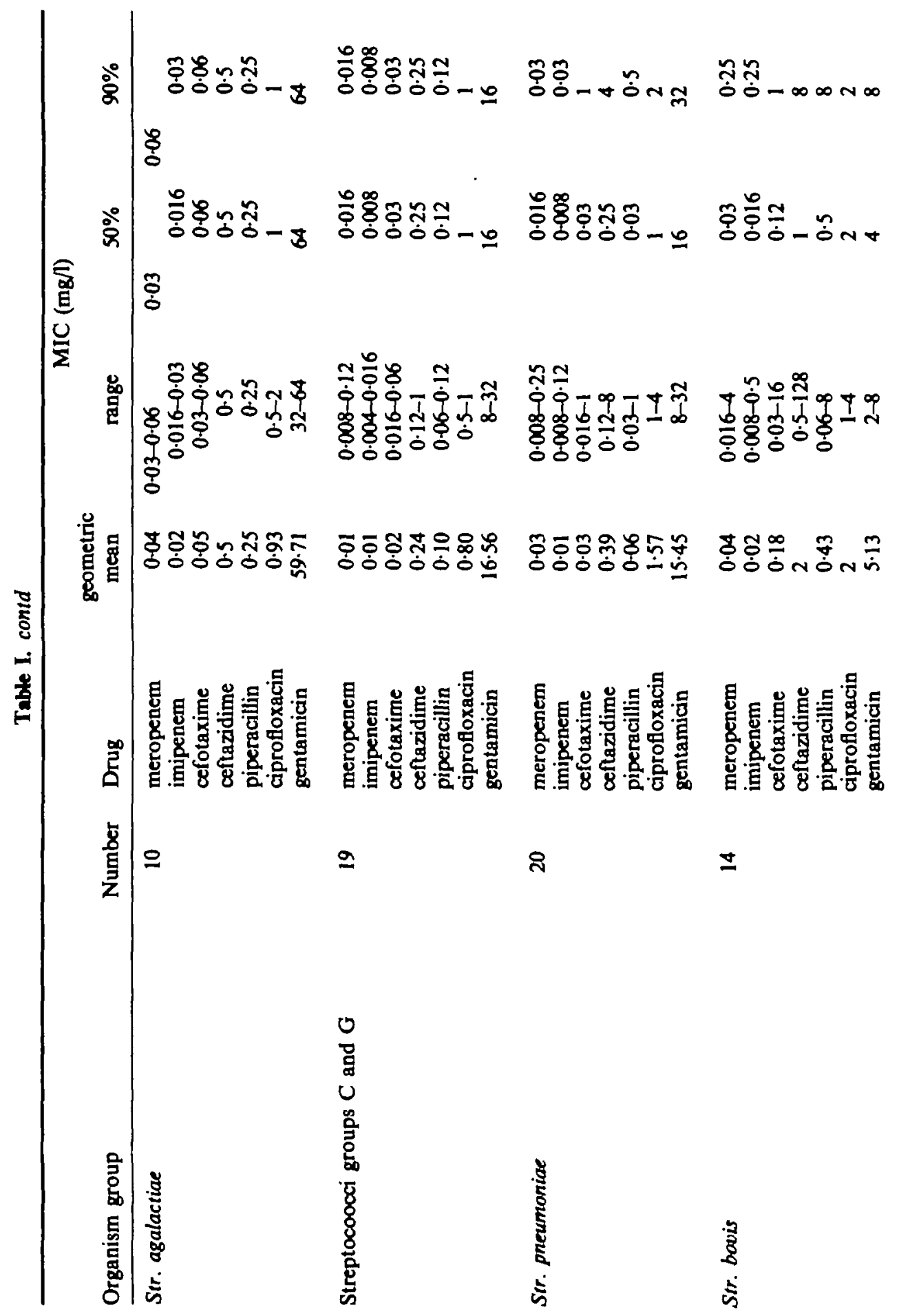




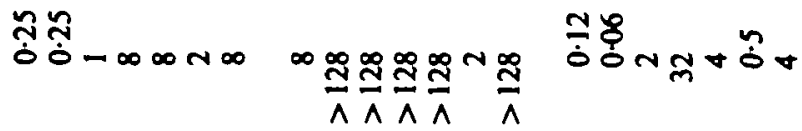

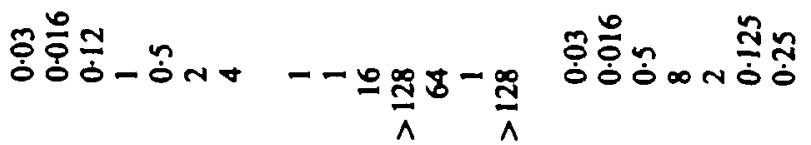

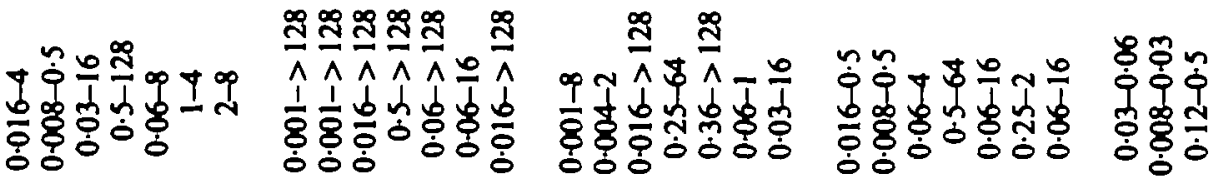

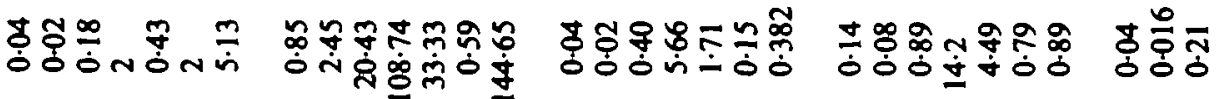

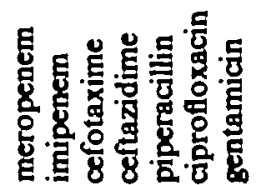

జ

$=$
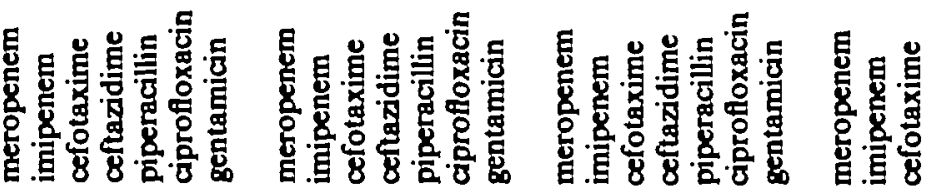

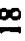

o

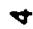

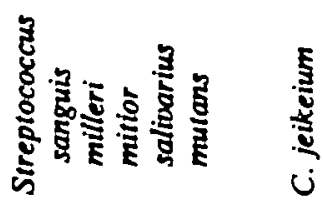

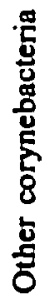




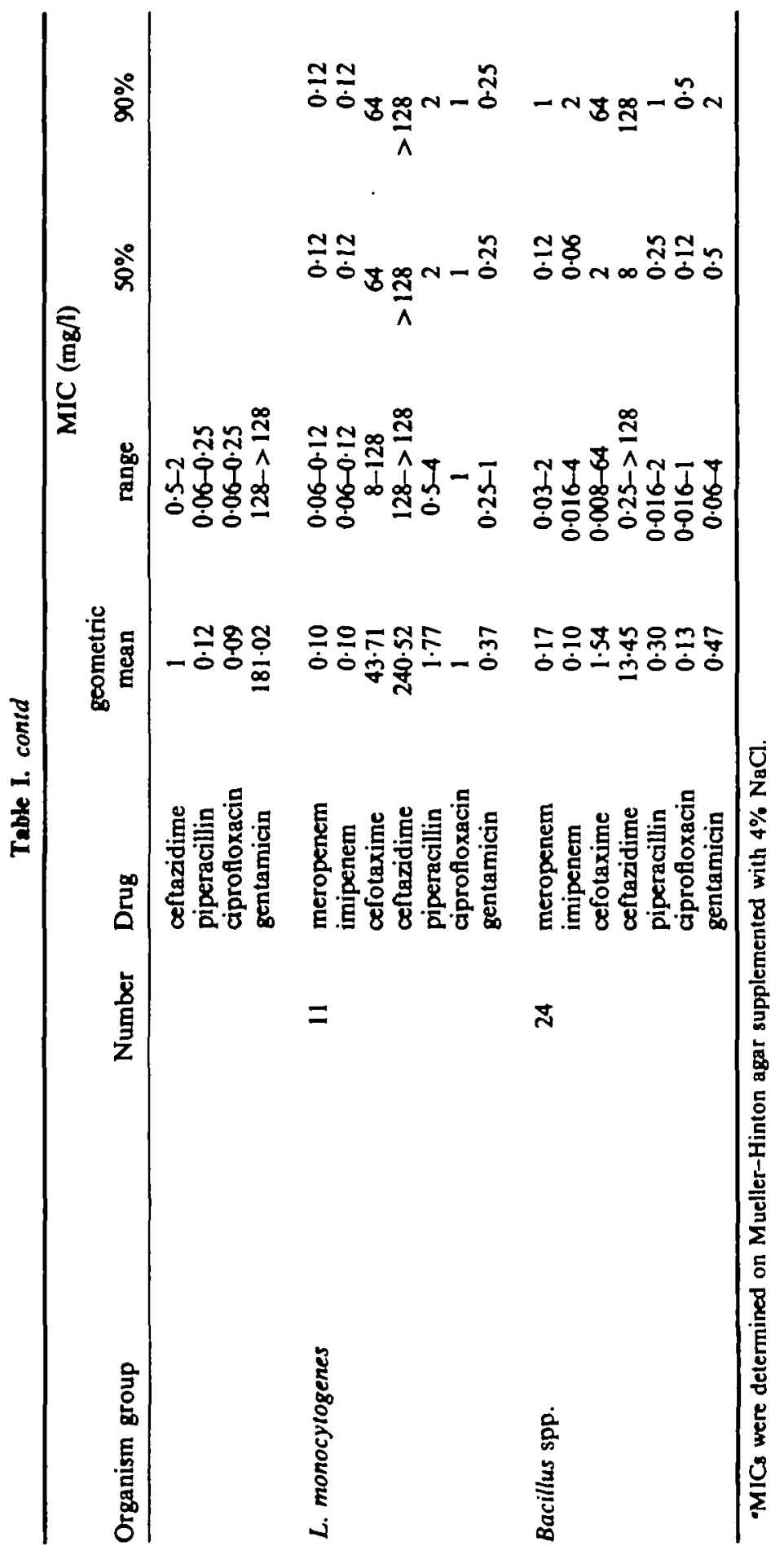




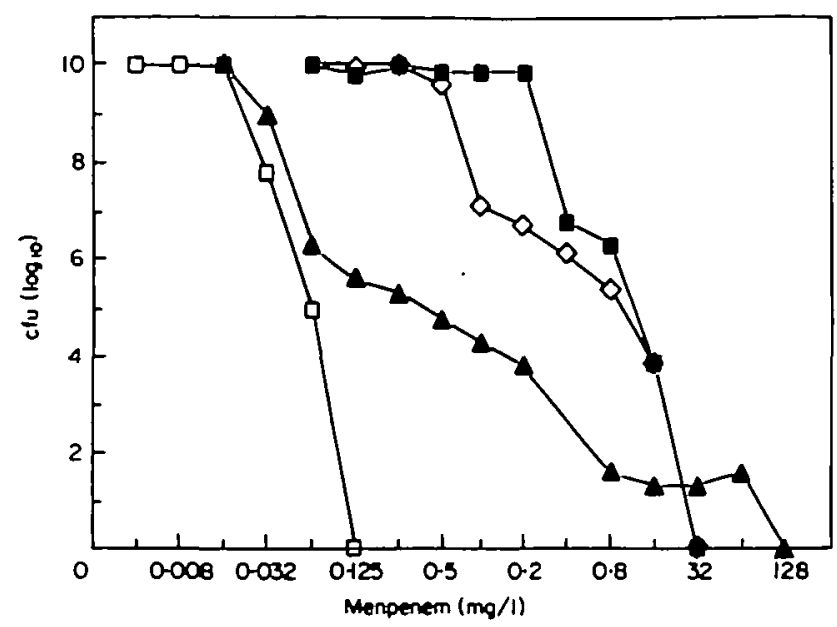

Figure 1. Population analysis of four isogenic Staph. aureus strains on meropenern. $\square$, Methicillinsusceptible strain BB255; $\square$, methicillin-resistant strain BB270; $\Delta$, methicillin-susceptible strain BB308 derived from BB270 by TnS51 insertion; $\diamond$, methicillin-resistant revertant strain $\mathrm{r} 2$ derived from BB308, with a deletion within the chromosome.

methicillin resistance determinant mec, had lost the expression of low level resistance and the high level resistance was impaired. Strain $\mathrm{r} 2$ was a revertant from BB308 with a deletion within the chromosome. This strain had regained both resistance levels and showed a resistance pattern similar to BB270. (For a more detailed description of the strains see Berger-Bächi et al., 1986.) The patterns shown in Figure 1 are very similar to results of population studies with imipenem (Berger-Bächi et al., 1986). Heteroresistance to meropenem was also observed in strains of coagulase-negative staphylococci harbouring the mec-determinant (data not shown). The results obtained by measuring the affinity of meropenem and imipenem to the PBPs of Staph. aureus were in accordance with the population studies (Figure 2). Whereas PBPs of the susceptible strain BB255 were saturated by low amounts of the antibiotics (Figure 2(a)), high amounts were necessary to saturate PBP 2', the biochemical correlate of methicillinresistance, in strain BB270 (see Figure 2(b)).

\section{Bactericidal activity of meropenem}

Examination of the bactericidal activity of meropenem and comparative agents initially was carried out by conventional MBC tests performed in triplicate. Although great care was taken in performing these tests, results were often inconsistent, especially with staphylococci, owing to methodological problems (see Taylor et al., 1983). We therefore decided to study the bactericidal potential of the drug in a kinetic system. Figure 3 shows the killing curves obtained with meropenem at various multiples of the MIC against three representative strains (one each of Staph. aureus, Streptococcus agalactiae and Enterococcus faecalis). The drug killed efficiently, even at rather low concentrations. The rate and the degree of killing of the Staph. aureus was less pronounced at four and eight times than at one and two times the MIC (Figure 3(a)). This well-known paradoxical effect was also observed with imipenem and flucloxacillin (data not shown). Not all the staphylococcal strains examined, however, exhibited this beha- 

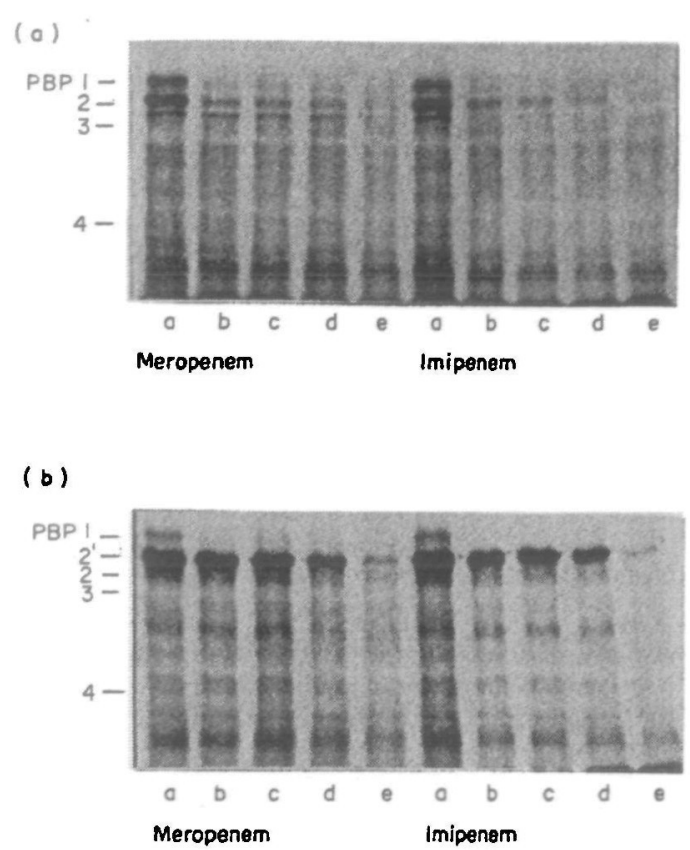

Figure 2. Fluorograph of penicillin binding proteins of (a) methicillin-susceptible Staph. aureus BB255 and (b) methicillin-resistant strain BB270. Membrane preparations were prepared as described and preincubated with various concentrations of either meropenem or imipenem, before treatment with ${ }^{3} \mathrm{H}$-labelled penicillin. Lanes: $a, 0 ; b, 0.5 ; c, 5 ; d, 50$; and e, $500 \mathrm{mg} / \mathrm{l}$ of either meropenem or imipenem, respectively.

viour. Figure 4 shows the killing curves of meropenem in comparison with imipenem and further bactericidal antimicrobials at four times the MIC of each antibiotic for Ent. faecium, C. jeikeium, and L.monocytogenes. The bactericidal activities of the $\beta$ lactam antibiotics tested were approximately equal. The rate of killing of meropenem and comparative drugs for all species was low during $6 \mathrm{~h}$ of incubation, but increased by incubating for $24 \mathrm{~h}$.

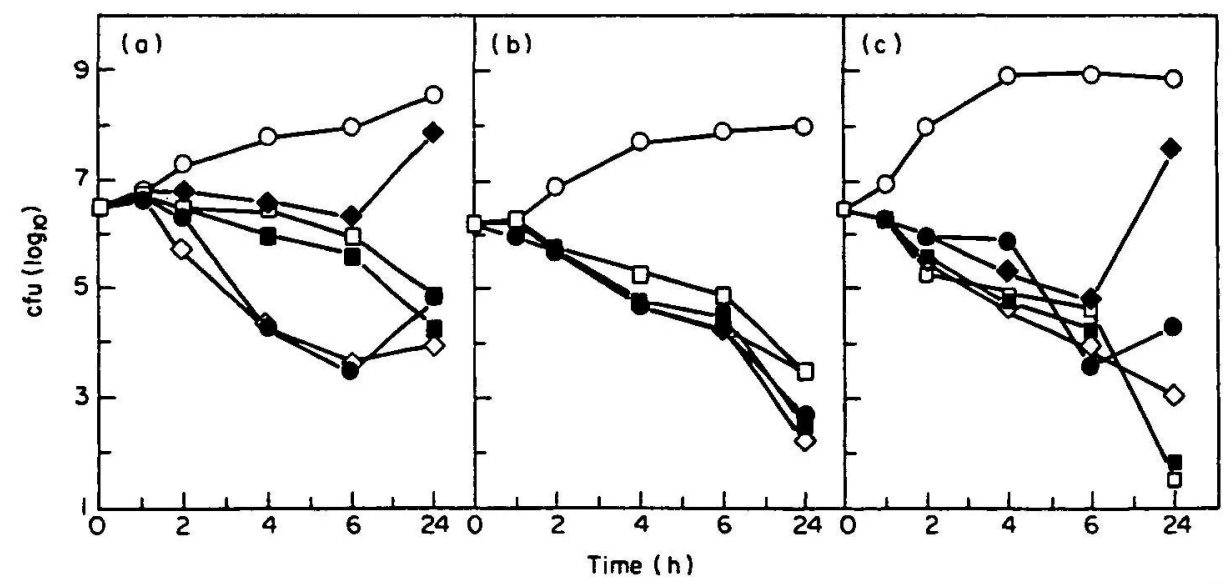

Figure 3. Rates of killing of (a) penicillinase-producing Staph. aureus (MIC of meropenem $0.25 \mathrm{mg} / \mathrm{l}$ ); (b) Ent. faecalis (MIC $4 \mathrm{mg} / \mathrm{l}$ ); (c) Str. agalactiae (MIC $0.016 \mathrm{mg} / 1$ ), by various concentration of meropenem. $O$, Control; $\bullet, \frac{1}{2} \times \mathrm{MIC} ; 0,1 \times \mathrm{MIC}, \diamond, 2 \times \mathrm{MIC}$; $4 \times \mathrm{MIC} ; \square, 8 \times \mathrm{MIC}$. 


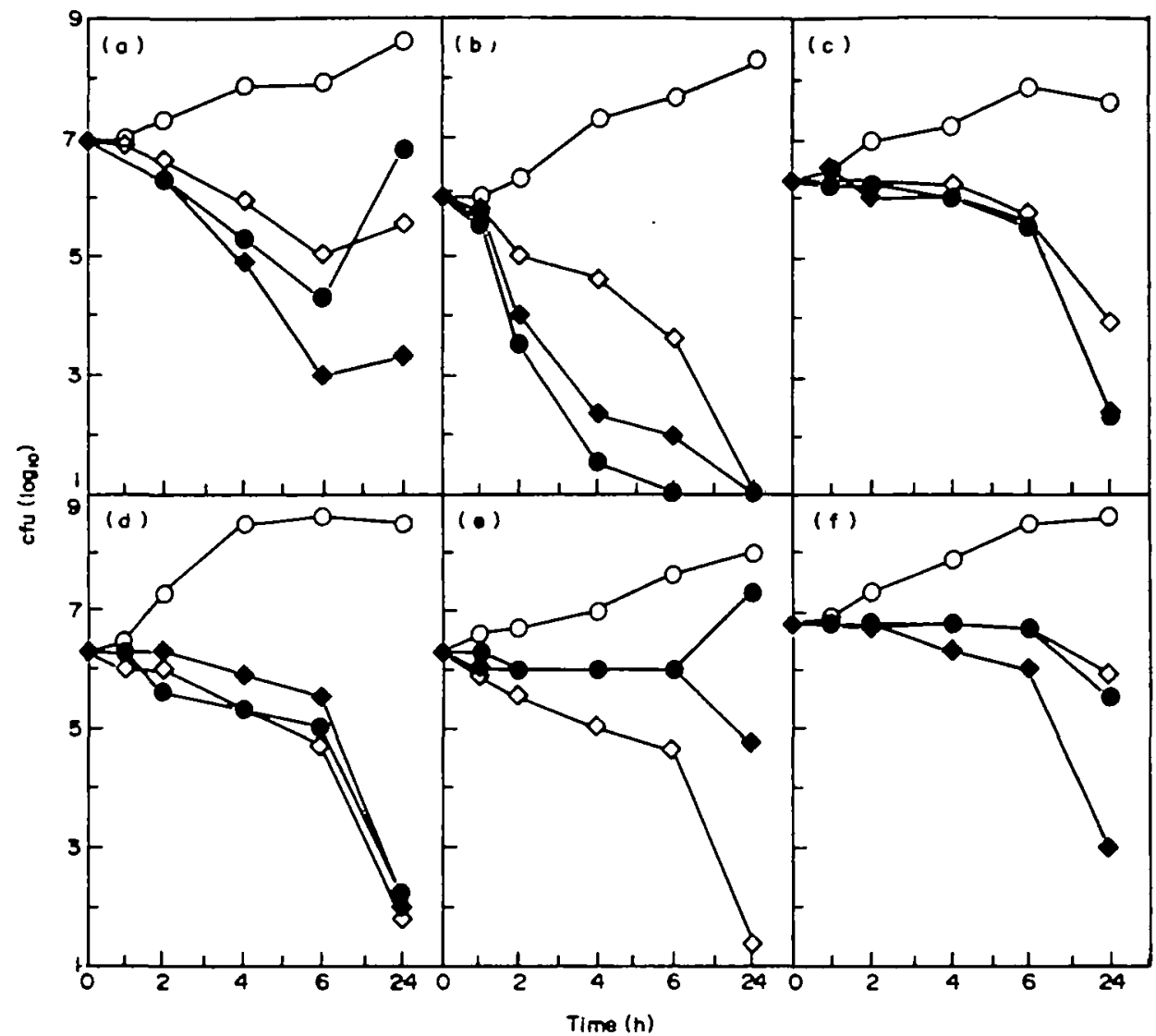

Fipure 4. Comparison of the rate of killing of Gram-poxitive organisms by 4 times the MIC of $\bullet$, meropenem; $O$, imipenem; $\diamond$, various bactericidal antimicrobials. $O$, Growth control. (a) Penicillinasenegative Staph. aureus (MIC of meropenem 0-06; imipenem 0-008; flucloxacillin (O) 0-25 mg/l). (b) Staph. epidermidis (MIC of meropenem 0-12; imipenem 0-03; vancomycin ( $\diamond) 2 \mathrm{mg} / \mathrm{h}$ ). (c) Ent. faecium (MIC of meropenem 4; imipenem 1; vancomycin (O) $4 \mathrm{mg} /$ ). (d) Strep. pyogenes (MIC of meropenem 0-016; imipenem 0-004; benzylpenicillin ( $)$ ) $0-016 \mathrm{mg} / 1$. (e) C.jeiketum (MIC of meropenem 2; imipenem 8; vancomycin (O) $1 \mathrm{mg} / \mathrm{I}$ ). (f) L. monacylogenes (MIC of meropenem 0-06; imipenem 0-06; ampicillin ( $(\Delta)$ $0.25 \mathrm{mg} / \mathrm{l}$ ).

\section{Discussion}

Meropenem was shown to be an excellent antibiotic against most species of Grampositive bacteria. In particular, the drug exhibited some activity against methicillinresistant staphylococci, when tested in agar dilution with an inoculum of $10^{4} \mathrm{cfu}$ per spot. However, the typical characteristics of methicillin-resistant staphylococci, namely heteroresistance (Figure 1), and the influence of incubation temperature, duration of incubation, and sodium chloride content of the agar medium on the phenotypic expression of resistance (data not shown), were also observed with meropenem (data not shown). Thus, as with all other $\beta$-lactam antibiotics (Kayser, 1980), meropenem does not seem likely to be a useful agent in the treatment of diseases caused by methicillin-resistant staphylococci. Meropenem exhibited high activity against all streptococcal species examined. Against Enterococcus faecalis, meropenem was approximately four times less active than imipenem, but showed similar activity to piperacillin, 
whereas, as expected, the cephalosporins were not active at all. Meropenem was as bactericidal as other $\beta$-lactam agents against most of the examined organisms in kinetic studies. Only poor bactericidal activity of the $\beta$-lactams was observed against Ent. faecium, C. jeikeium and L. monocytogenes.

In summary, this new experimental carbapenem antibiotic exhibited nearly identical properties to imipenem against Gram-positive organisms. Its greater stability to the dehydropeptidase-1 enzyme of the human kidney and its superior activity against Gram-negative organisms, including Pseudomonas spp., justify clinical trials.

\section{References}

Berger-Bächi, B., Strässle, A., \& Kayser, F. H. (1986). Characterization of an isogenic set of methicillin-resistant and susceptible mutants of Staphylococcus aureus. European Journal of Clinical Microbiology 5, 697-701.

Gill, V. J., Manning, C., Lamson, M., Woltering, P. \& Pizzo, P. A. (1981). Antibiotic-resistant group JK bacteria in hospitals. Journal of Clinical Microbiology 13, 472-7.

Jorgensen, J. H. (1986). Laboratory and epidemiologic experience with methicillin-resistant Staphylococcus aureus in the USA. European Journal of Clinical Microbiology 5, 693-6.

Kayser, F. H. (1980). Die Resistenz methicillinresistenter Staphylokokken gegenüber neuen Cephalosporin-Antibiotika. Infection 8, 165-70.

Kayser, F. H., Benner, E. J., Troy, R. \& Hoeprich, P. D. (1972). Mode of resistance against $\beta$ lactam antibiotics in staphylococci. Annals of the New York Academy of Sciences 182, 10617.

National Committee for Clinical Laboratory Standards (NCCLS) (1985). Methods for dilution antimicrobial susceptibility tests for bacteria that grow aerobically. Approved Standard M7$A$. The National Committee for Clinical Laboratory Standards, Vol. 5, No. 22.

National Committee for Clinical Laboratory Standards (NCCLS) (1987). Performance standards for antimicrobial susceptibility testing. First informational Supplement M7-A-S2. The National Committee for Clinical Laboratory Standards, Vol. 7, No. 10.

Pierard, D., Lauwers, S., Mouton, M. Sennesael, J. \& Verbeelen, D. (1983). Group JK corynebacterium peritonitis in a patient undergoing continuous ambulatory peritoneal dialysis. Journal of Clinical Microbiology 18, $1011-4$.

Taylor, P. C., Schoenknecht, F. D., Sherris, J. C. \& Linner, E. C. (1983). Determination of minimum bactericidal concentration of oxacillin for Staphylococcus aureus: influence and significance of technical factors. Antimicrobial Agents and Chemotherapy 23, 142-50.

Young, V. M., Meyers, W. F., Moody, M. R. \& Schimpff, S. C. (1981). The emergence of coryneform bacteria as a cause of nosocomial infection in compromised hosts. American Journal of Medicine 70, 646-50. 